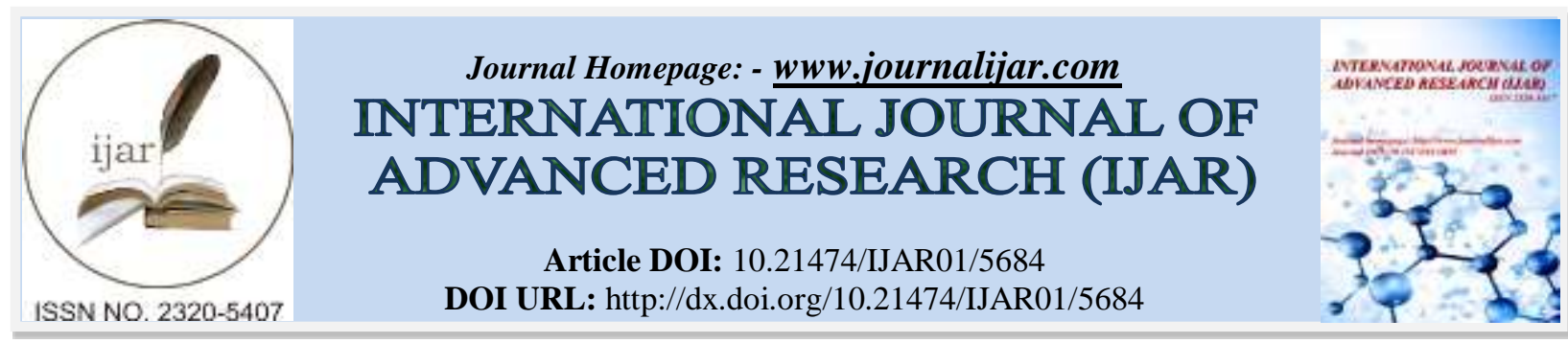

RESEARCH ARTICLE

\title{
LINE OF SIGHT VISIBLE LIGHT COMMUNICATION - AN INDOOR MODEL DESIGN AND ANALYSIS.
}

Nijanth Anand and Prof R. Suresh.

\section{Manuscript Info}

Manuscript History

Received: 21 August 2017

Final Accepted: 23 September 2017

Published: October 2017

Key words:-

VLC - Visible Light Communication;

Li-fi - Light Fidelity; LED - Light

Emitting Diode.

\begin{abstract}
Visible light communication or light fidelity also referred to as li-fi technology is the latest upcoming technology in the communication segment with it's innumerable advantages like security, safety, availability etc providing unimaginable high speed data connectivity possibilities. In this project we are going to realize a visible light communication system which is capable of detecting if a person is present and starts transferring data to him when he is in a stipulated range on the li-fi system by using visible light communication.
\end{abstract}

Copy Right, IJAR, 2017,. All rights reserved.

\section{Introduction:-}

Transferring data from one place to another is one of the key day-day affairs in today's world and hence achieving High Data rates has become a very important necessity. The whole bandwidth of the Visible Light between 400 and $800 \mathrm{THz}(780-375 \mathrm{~nm})$ if tapped can provide high speeds which is the core concept of VLC. Harald Haas, a wireless professor in the University of Edinburgh is widely considered to be the inventor of this Li-Fi Technology. Haas was the first person to coin the name Li-Fi in his TED Global Talk on Visible Light Communication were he successfully transmitted a High Definition Video. This emerging Communication Technology not only provides communication and illumination but also high security as the Light waves used in a closed room can't penetrate through walls like Radio waves ${ }^{[9]}$. VLC has been standardized as IEEE 802.15.7 by the Institute of Electrical and Electronics Engineers (IEEE) ${ }^{[8]}$. VLC Technology can be used in places like Airplanes, Hospitals were Radio waves aren't safe to use in comparison with Wi-fi Technology.

The basic Concept behind VLC is the Laser Emitting Diode(LED) at the Transmitter end transmits 1's and 0's by flickering the LED On or Off ${ }^{[3]}$. Since the flickering rate of the LED is very high It can't be perceived by the human eye $^{[4]}$. Audio, Video and Files can be transferred easily by encoding and passing through the LED driver which in turn is connected to the LED. The light ray's reach the Receiver, preferably a Silicon Photo-Diode having good response to visible light ${ }^{[3]}$. The light signals received at the receiver can be demodulated and decoded to get the data. Line-Of-Sight propagation is generally preferred because in the case of Multipath Propagation, there will be decrease in the efficiency ${ }^{[2]}$.

\section{Modulation Schemes:-}

Different modulation schemes are used in practice and are discussed in this paper. On Off $\mathrm{Keying}(\mathrm{OOK})$ is the easiest and the most basic modulation scheme which uses ON for 1's and OFF for 0's ${ }^{[1]}$. In OFF state it is not necessary that the intensity has to be zero, instead the intensity can be decreased. Data rates of $100 \mathrm{Mbits} / \mathrm{s}$ can be achieved using OOK, but in the case of Multipath Propagation delay spread produces Inter Symbol Interference(ISI) resulting in data rates of $10 \mathrm{Mbits} / \mathrm{s}$ requiring additional receiver complexity ${ }^{[11]}$. Variable Pulse Position 
Modulation(VPPM) does the encoding by varying the duty cycle(Pulse Width) of the optical symbol. Change in duty cycle (Pulse Width) has to be done in response to the required dimming level. So logic's 0 and 1 are given Pulse Widths depending on the required dimming level using Pulse Width Modulation(PWM) like 75 percent dimming duty cycle requirement. The preamble and header are always at 50 percent duty cycle and hence adjustments in compensation time is required to keep the brightness constant which may not be possible in large dimming ranges ${ }^{[1]}$. Color Shift keying(CSK) is used in multicolor RGB LED's similar to Frequency Division Multiplexing(FDM) were different light color's are used instead of frequencies providing high data rates at low clock frequencies. Discrete Multi Tone(DMT) Modulation can be used to achieve data rates as high as $1 \mathrm{~Gb} / \mathrm{s}^{[10]} \mathrm{by}$ decomposing a signal into multiple sub-carriers and performing IFFT in the Transmitter alongside cyclic prefix and binary data mapping. Orthogonal Frequency Division multiplexing(OFDM) is also used widely, in combination with multiple access schemes such as Time Division Multiplexing(TDM) and Frequency Division Multiplexing(FDM) providing high bandwidth. Multipath induced ISI is mitigated effectively in OFDM alongside simple equalization at the receiver making the practical implementation easier ${ }^{[11]}$.

\section{Applications Of Visible Light Communication:-}

VLC has plenty of applications ranging from smart lighting in smart buildings were illumination control and communication will help save energy to underwater communications were RF doesn't work. In Hazardous environments like Oil Rigs, Mines, Petrol Bunks were there is risk of explosions, VLC provides safe communication. Also by pointing visible light on a device you can achieve data transfer without having to pair or connect therby making the process simpler and faster owing to the high data rates compared to Wi-fi and Bluetooth. VLC finds use in defence and security as the light waves can't be detected on the other side of the wall ${ }^{[5]}$.

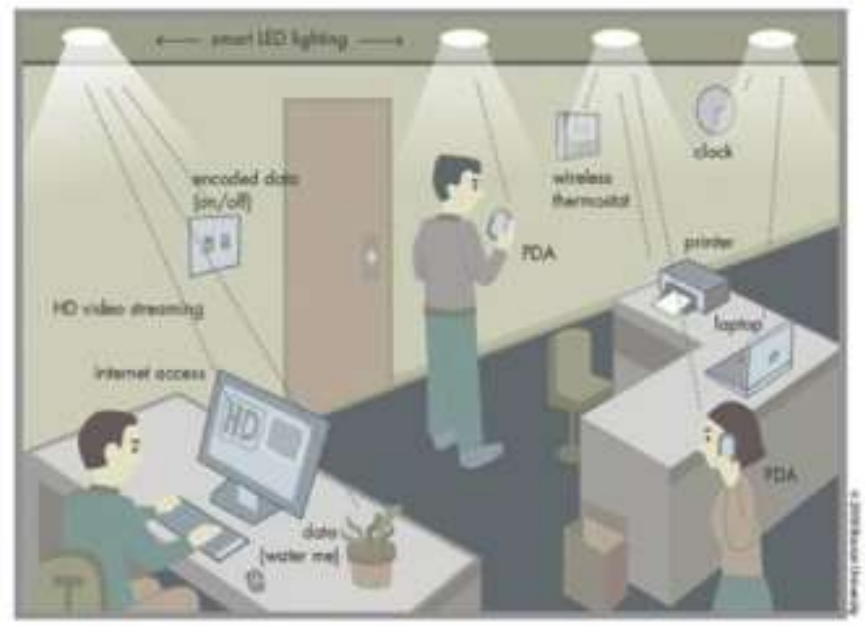

Figure 1 :-Applications of Visible Light Communication

In our project we stick to a specific use of Visible Light Communication for transferring data from a Computer to a User via a Mobile Phone. We use a PIR Sensor to detect the presence of human interference and if there is any human interference then the text data is communicated to the user using Visible Light Communication. This designed application of VLC finds use in the corridors of the classrooms in VIT, every time a seminar or a workshop is happening inside campus no one is aware of it. So using this system anyone crossing through it will be notified of the happenings around him. Also in Supermarkets once the user enters a particular section he gets all the details and offers in the products in that section.

\section{Design Of The Vlc System:-}

The newly proposed system consists of a Li-fi transmission system that is capable of transmitting text data from a Computer to a Mobile Phone. So the entire system is divided into two segments the Transmitter and the Receiver segment both having many identical components with varying functionalities.

The Transmitter Segment receives the Input from the Laptop as Text data and transmits it as text data. The block diagram of the Transmitter segment is shown below and then it is discussed in detail. 


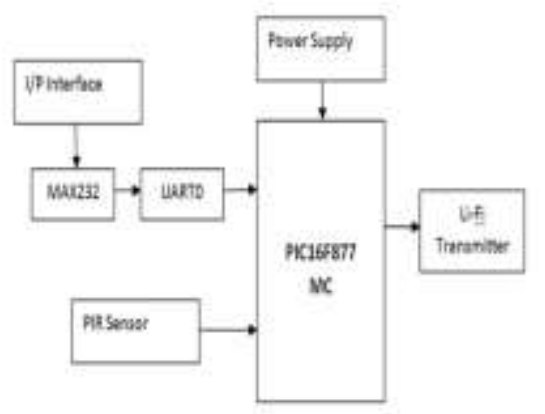

Fig 2:- Block diagram of the Li-fi Transmitter

The I/P Interface is the first block which refers to the I/P interfacing from the Laptop, the text data from the Laptop is the I/P interface for the proposed Li-fi System. Serial data from the Laptop is received by using the Hyperterminal Window which receives the Input from the Keyboard or via the On Screen Keyboard in the Laptop. The Hyperterminal window connects with the port in which the UART is connected based on how it is configured thereby providing I/P interface. The signal from the I/P interface is displayed on the LCD in the Microcontroller. The UART module is connected to the Laptop and is used to convert Serial data into Parallel Data. The UART specified over here is an USB to RS232 Module which can be connected to the USB port of the Laptop and interfaced with the RS232 module giving parallel data input to the system. So the UART ensures data transfer from the I/P interface to the System.

The TTL232 acts as a bridge between the UART and the Microcontroller. As we know the data from the Computer is of Resistor Transistor Logic and hence has voltages ranging from 0 to $10 \mathrm{~V}$ whereas a Microcontroller can follow only Transistor Transistor Logic which involves Voltages from 0 to 5V. So the TTL232 converts all the digital logics from RTL format into TTL format.

Power Supply is another crucial component which is required to provide $5 \mathrm{~V}$ constant power Supply to the Microcontroller for it's functioning. It consists of a $230 \mathrm{~V}$ AC supply which is converted to DC using Diodes followed by filtering using Capacitance and then a 5805 Voltage Regulator which converts it to 5V DC. The power supply is present both at the Transmitter and the Receiver end of the Microcontroller.

The Passive Infrared Sensor otherwise referred to as a PIR Sensor detects human Interference. The PIR Sensor has 3 ports associated with it of which two are for Power Supply and the other is the Output wave. If there is any human interference then the PIR sensor detects it and transmits some voltage ranging from 3.3 to $5 \mathrm{~V}$ if there is any human interference. If there is no human interference then no Voltage is transmitted. This check by the PIR Sensor happens every 3 seconds.

The Microcontroller is the heart of the system and is responsible for the entire functionality of the system. The microcontroller chosen over here is a PIC16F877A microcontroller. The microcontroller is responsible for the reception of the Text data and for changing the formats of the data for it's transmission based on the input from the PIR Sensor. It plays a crucial role and follows the Star Hash Algorithm which is discussed.

The Final component of the System is the Li-fi transmitter which receives the data from the microcontroller and transmits it as Visible Light via the Light Source. The Analysis of the Transmitting Light Source has been performed in the Chapters that follow. The Li-fi transmitter is the final stage of the transmission with the Light Source operating at a Voltage of $9 \mathrm{~V}$.

The Receiver End of the System also plays a crucial role as it is responsible for reception of the signal and transmission to the Mobile Phone. The block diagram of the Receiver Circuit is shown below. 


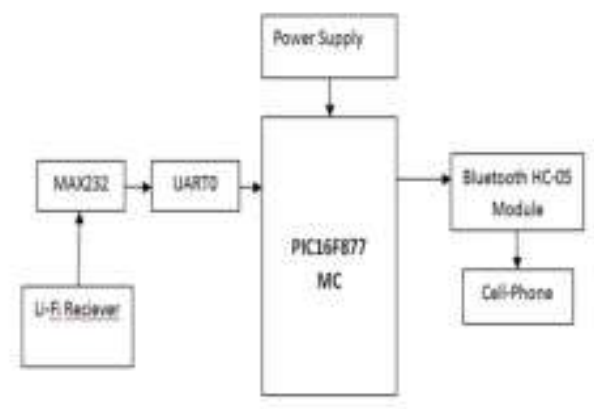

Fig 3:- Block Diagram of the Receiver Circuit.

The Receiver block also has 7 components associated with it with changes in functionality and components compared to that of a Transmitter. It is responsible for reception of the data and interpreting the Text data and displaying it on the Cell phone and the LCD. The technical aspects of the components in the receiver are discussed in the next chapter. The functionality of each of the components is discussed below.

The Li-fi Receiver is the first component which comprises of an IR Sensor which detects the signal and sends it to the Microcontroller via the UART. The Receiver can be held at a distance of 80-100 metres from the Transmitter an analysis of which is present in the following Chapters. The Technical aspect of the Li-fi Receiver has been discussed in the next chapter. The Receipted signal is displayed on the LCD in the Microcontroller.

The decoded signal from the Li-fi Receiver is fed to the Microcontroller. The microcontroller receives the data and intercepts it using the "Star and Hash Algorithm" and displays the text data on the LCD in the microcontroller. The Microcontroller also has the Bluetooth HC-05 module connected at it's Transmitter port. So it transmits the signal to the transmitter port after the reception of the signal and it's correct interception using the "Star and Hash Algorithm".

The BluetoothHC-05 module is responsible for establishing connectivity with the mobile phones which is restricted to phones operating on Android operating system. The Bluetooth Module receives the data from the Transmitter end of the Microcontroller and transmits it to all devices paired to the Bluetooth Module. It consists of three ports, two for power supply and one which connects the Microcontroller to the Module from the Transmitter end of the Microcontroller resulting in data transmission.

The Cell phone is the last stage of the Receiver were in the data has to be displayed on the screen. So we have to install applications such as BlueTerm or TerminalBt which are capable of receiving data once connected to the Bluetooth module. So once the pairing is done via Bluetooth settings the data can be received in the mobile phone establishing Li-fi based data communication.

The power supply used at the Receiver end is similar to the Power Supply used at the Transmitter end with no changes to it.

\section{THE STAR and HASH Algorithm:-}

The data received from the computer is continuous and you can't predict when it starts and when it ends. So we come up with an algorithm to figure out when the word starts and ends so that we can get the exact word typed at the I/P Interface. So as per the Algorithm we are supposed to start each statement with a star "** and then type in whatever Word we want to Communicate and end it with a Hash “\#”.

It is to be noted that the Microcontroller programming primarily involves interfacing the Input and Output devices like the PIR sensor, Bluetooth Module and the Li-fi Transmitter and Receivers. But apart from all this it implements the Star and Hash Algorithm logic. The data is given at the Input Interface using the Star and Hash logic and the logic for it's processing has to be dealt with in the microcontroller. The algorithm for it's implementation is given below. 
Initiate Flag as 0 which is a reference variable:-

1. Check if the character is a* as it indicates the start of a word.

2. If it starts with $\mathrm{a} *$ then initiate Flag as 1 .

3. If Flag is 1 then check the variable.

(a)If it is not a \# then it is a part of the word and it is printed and transmitted to the Rx.

(b)If it's a \# then the Word has ended and hence we disable the interrupt and set the value of flag as 2.

4. We check the value of flag. If it is 2 then it is set back as 0 and sent to Step 1 . If the value of flag is 1 then it keeps iterating with Step 4.

The same algorithm is common for both the Transmitter and the Receiver as in both cases the logic is same with only the Tx and Rx components varying.

\section{Analysis Of The Vlc System:-}

The Li-fi system has successfully transmitted data and the end result has been obtained, also the analysis of the system has been performed taking into consideration various aspects.

\section{Line Of Sight At Different Environments:-}

It has been found that the Line of Sight of the Li-fi system keeps on varying based on the environment. For example the Line of Sight is found to be high in a completely dark room when compared to that of a room with high illumination. An experimental analysis was performed to measure the line of sight at different atmospheric constraints and the results are shown below.

\begin{tabular}{|c|c|c|}
\hline \multirow{2}{*}{ Condition } & \multicolumn{2}{|c|}{ Line Of Sight Distance } \\
\cline { 2 - 3 } & Avg & Max \\
\hline $\begin{array}{c}\text { Daytime - No } \\
\text { interference }\end{array}$ & $125 \mathrm{~cm}$ & $150 \mathrm{~cm}$ \\
\hline $\begin{array}{c}\text { Daytime - With } \\
\text { interference }\end{array}$ & $80 \mathrm{~cm}$ & $110 \mathrm{~cm}$ \\
\hline Night Time & $60 \mathrm{~cm}$ & $90 \mathrm{~cm}$ \\
\hline Complete Darkness & $180 \mathrm{~cm}$ & $200 \mathrm{~cm}$ \\
\hline
\end{tabular}

Fig 4:- Line of Sight at different Conditions

As you can see from the above table the Line Of Sight was calculated at different atmospheric constraints in the laboratory. Initially the readings were taken in the laboratory without any interference with minimal amount of lighting and it was found to average $125 \mathrm{~cm}$. The readings were then taken in the Laboratory with Interference that is with all the lights on and we found some decrease in the Line Of Sight because of External Light Interference and was $80 \mathrm{~cm}$. The third set of readings was taken at night with no interference from Sunlight and only light from other sources. A further decrease in the line of sight was witnessed in this case making it $60 \mathrm{~cm}$. The final set of readings were taken in complete darkness and it was found to have the highest Line of Sight Distance because of Zero Interference from other sources giving it a maximum LOS distance of $180 \mathrm{~cm}$.

\section{Light Intensity at Different Data Rates:-}

The data rates are varied by changing the baud rates and checking the intensity at different Baud rates using the Lux Meter. The tabular column for the Light Intensities at different Data Rates is shown below. 


\begin{tabular}{|c|c|c|c|}
\hline \multirow{2}{*}{ Baud Rate } & \multicolumn{3}{|c|}{ Intensity (Lx) } \\
\cline { 2 - 4 } & Min & Max & Avg \\
\hline 2400 & 106 & 120 & 113 \\
\hline 4800 & 106 & 124 & 115 \\
\hline 9600 & 120 & 134 & 127 \\
\hline 19200 & 124 & 138 & 131 \\
\hline
\end{tabular}

Fig 5:- Intensity Vs Baud Rate

The experiment was performed in the laboratory in Daytime with No Interference. The experimental Setup shows that the Light Intensity increases as the Baud Rate increases because the Data Rate increases. Thereby it is found that the Light Intensity is proportional to the baud rate as the Light Intensity is high when the Baud rate is high and vice versa.

\section{Transmission In a Medium With No Interference:-}

An analysis of the system is performed in a closed atmosphere to observe the changes in the line of sight distance observed when compared to the line of sight distance in an ideal atmosphere. We choose a PVC pipe as a medium with no outside interference in this case

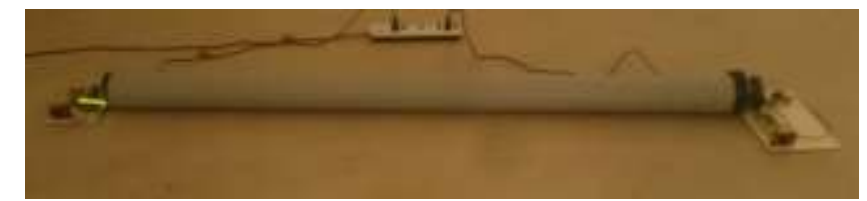

Fig 6:- Experimental Setup for the Analysis using a PVC pipe.

The above experiment was performed and the Line Of Sight distance was found to be $300 \mathrm{~cm}$ close to which was way more than the usual Line Of Sight Observed and hence proved that the performance increased when there was no interference from other outside sources.

Also it was observed that the performance of the system was enhanced as there were very little errors in the Receiver end because in the usual conditions there is beam dispersion, atmospheric absorption, shadowing, pollution etc which results in an attenuated receiver signal that results in a higher Bit Error Rate (BER).

\section{Voltage Level At The Transmitter And The Receiver:-}

At the transmitter end, the PIR Sensor detects every 3 seconds and only based on what the PIR Sensor detects, the data is transmitted. A square wave has been found to be generated at the Transmitter end with a Voltage of 5.2V and having a Frequency of $3.133 \mathrm{Khz}$. The digital Square wave with a fixed voltage and frequency is generated only every 3 seconds apart from which only noise is transmitted.

The output from the Lifi Receiver which is given as input to the Microcontroller at the $\mathrm{C} 7$ port is analyzed using the Oscilloscope. As you can see Square waves are generated with a Voltage of 5.21V and frequency of $1.5626 \mathrm{Khz}$. Even the waveform at the Receiver end is found to occur only at intervals of 3 seconds because of the delay in sensing by the PIR Sensor.

\section{Challenges:-}

The response of the VLC system can be increased by transmitter and/or receiver equalization, or complex modulation schemes and by using parallel communication were in many LED's are used at the transmitter and a receiver array is used making it a optical MIMO(Multiple Input Multiple Output) optical system ${ }^{[6]}$. There is a difference in data rates between an empty room and an office room which is filled with objects resulting in great multipath propagation and delay spread which is a major challenge in the VLC system ${ }^{[7]}$. Human inference like walking in the room will have an effect on the efficiency of the VLC system that is the Line of Sight Distance. 


\section{Conclusion:-}

In this paper, the core fundamentals of Visible Light Communication have been discussed along with the different modulation schemes and a VLC system has been realized that is capable of communicating between the Laptop and the Cell phone by detecting if there is any Human Interference. Also an Analysis of the Li-fi system is performed successfully. Thereby in future VLC is bound to play a very important role in everyone's life with it's unbound capabilities.

\section{References:-}

1. Sridhar rajagopal, samsung electronics richard d. Roberts, intel sang-kyu lim, etri(2012), ieee 802.15.7 visible light communication: modulation schemes and dimming support, ieee communications magazine , march 2012

2. Line-of-sight visible light communication system design and demonstration, kaiyum cui ; dept. Of electr. Eng., univ. Of california, riverside, ca, usa ; gang chen ; zhengyuan xu ; roberts, r.d. Ieee(2010)

3. RAHUL R. SHARMA, RAUNAK, AKSHAY SANGANAL(2014), LI-FI TECHNOLOGY TRANSMISSION OF DATA THROUGH LIGHT, INT.J.COMPUTER TECHNOLOGY \& APPLICATIONS, VOL 5 (1),150-154

4. Sathiya.t, prof.e.divya, prof.s.raja(2014), visible light communication for wireless data transmission international journal of innovative research in electrical, electronics, instrumentation and control engineering vol. 2, issue 2 , february 2014

5. Rajan sagotra and reena aggarwal (2013), visible light communication, international journal of computer trends and technology (ijctt) - volume4issue4 -april 2013

6. Dominic c. O'brien, lubin zeng, hoa le-minh, grahame faulkner, joachim w walewski, sebastian randel(2008) ,visible light communications: challenges and possibilities, ieee journal

7. Ahmed taha hussein and jaafar m.h. Elmirghani(2015), performance evaluation of multi-gigabit indoor visible light communication system , $201520^{\text {th }}$ european conference on networks and optical communications, 30 jun -02 jul 2015

8. Ieee standard for local and metropolitan area networks -- part 15.7: short-range wireless optical communication using visible light. Pp. 1-309,september 2011. Http://www.huffingtonpost.com/entry/harald-haas-wirelessdata_n_1153538.html?1324050673=\&section=india

9. A. M. Khalid, g. Cossu, r. Corsini, p. Choudhury, and e. Ciaramella(2012), 1-gb/s transmissionoveraphosphorescent white led by using rate-adaptive discrete multitone modulation, ieee photonics journal, vol. 4, no. 5, october 2012

10. Hany elgala, raed mesleh, and harald haas(2009), indoor broadcasting via white leds and ofdm , ieee transactions on consumer electronics, vol. 55, no. 3, august 2009. 